\title{
State and Trait Components of Functional Connectivity: Individual Differences Vary with Mental State
}

\author{
Linda Geerligs, ${ }^{1,4}$ Mikail Rubinov, ${ }^{2,3}$ Cam-CAN, ${ }^{4}$ and Richard N. Henson ${ }^{1,4}$ \\ ${ }^{1}$ Medical Research Council (MRC) Cognition and Brain Sciences Unit, Cambridge CB2 7EF, United Kingdom, ${ }^{2}$ Brain Mapping Unit, Department of \\ Psychiatry, University of Cambridge, Cambridge CB2 3EB, United Kingdom, ${ }^{3}$ Churchill College, University of Cambridge, Cambridge CB3 0DS, United \\ Kingdom, and ${ }^{4}$ Cambridge Centre for Ageing and Neuroscience (Cam-CAN), University of Cambridge and MRC Cognition and Brain Sciences Unit, \\ Cambridge, United Kingdom
}

Resting-state functional connectivity, as measured by functional magnetic resonance imaging (fMRI), is often treated as a trait, used, for example, to draw inferences about individual differences in cognitive function, or differences between healthy or diseased populations. However, functional connectivity can also depend on the individual's mental state. In the present study, we examined the relative contribution of state and trait components in shaping an individual's functional architecture. We used fMRI data from a large, population-based human sample ( $N=587$, age 18 - 88 years), as part of the Cambridge Centre for Aging and Neuroscience (Cam-CAN), which were collected in three mental states: resting, performing a sensorimotor task, and watching a movie. Whereas previous studies have shown commonalities across mental states in the average functional connectivity across individuals, we focused on the effects of states on the pattern of individual differences in functional connectivity. We found that state effects were as important as trait effects in shaping individual functional connectivity patterns, each explaining an approximately equal amount of variance. This was true when we looked at aging, as one specific dimension of individual differences, as well as when we looked at generic aspects of individual variation. These results show that individual differences in functional connectivity consist of state-dependent aspects, as well as more stable, trait-like characteristics. Studying individual differences in functional connectivity across a wider range of mental states will therefore provide a more complete picture of the mechanisms underlying factors such as cognitive ability, aging, and disease.

Key words: aging; brain connectivity; fMRI; network; resting state

\section{Significance Statement}

The brain's functional architecture is remarkably similar across different individuals and across different mental states, which is why many studies use functional connectivity as a trait measure. Despite these trait-like aspects, functional connectivity varies over time and with changes in cognitive state. We measured connectivity in three different states to quantify the size of the trait-like component of functional connectivity, compared with the state-dependent component. Our results show that studying individual differences within one state (such as resting) uncovers only part of the relevant individual differences in brain function, and that the study of functional connectivity under multiple mental states is essential to disentangle connectivity differences that are transient versus those that represent more stable, trait-like characteristics of an individual.

\section{Introduction}

The brain's functional architecture is remarkably similar across different individuals and across different mental states (Calhoun et al., 2008; Smith et al., 2009; Cole et al., 2014; Krienen et al.,
2014). Regardless of the population that is studied and the methods that are used, the same functional networks emerge of brain regions that show high connectivity with each other (Damoiseaux et al., 2006; Power et al., 2011). Because functional connecassistance.

The Cam-CAN corporate author consists of the project principal personnel: Lorraine K. Tyler, Carol Brayne, Edward T. Bullmore, Andrew C. Calder, Rhodri Cusack, Tim Dalgleish, John Duncan, Richard N. Henson, Fiona E. Matthews, William D. Marslen-Wilson, James B. Rowe, Meredith A. Shafto; Research Associates: Karen Campbell, Teresa Cheung, Simon Davis, Linda Geerligs, Rogier Kievit, Anna McCarrey, Abdur Mustafa, Darren Price, David Samu, Jason R. Taylor, Matthias Treder, Kamen Tsvetanov, Janna van Belle, Nitin Williams; Research Assistants: Lauren Bates, Tina Emery, Sharon Erzinçlioglu, Andrew Gadie, Sofia Gerbase, Stanimira Georgieva, Claire Hanley, Beth Parkin, 
tivity appears to be such a stable characteristic of the brain, many studies now use functional connectivity as a trait measure. For example, individual differences in functional connectivity in resting state have been linked to individual differences in cognitive function, age, and mental health (van den Heuvel et al., 2009; Alexander-Bloch et al., 2010; Sanz-Arigita et al., 2010; Brier et al., 2014; Geerligs et al., 2015). Many of these studies have yielded valuable insights into the functional changes underlying a variety of abilities and deficits (see also Uddin et al., 2010).

However, despite these trait-like aspects, functional connectivity also has a state-dependent aspect. Functional connectivity has been shown to change with cognitive states and cognitive demand (Kitzbichler et al., 2011; Moussa et al., 2011; Shirer et al., 2012; Di et al., 2013; Mennes et al., 2013; Gonzalez-Castillo et al., 2015). Even within a resting-state scan, significant temporal fluctuations in functional connectivity can be uncovered (Hutchison et al., 2013; Allen et al., 2014; Zalesky et al., 2014). In addition, past experiences, as well as ongoing ones, have been shown to modulate the functional architecture (Barnes et al., 2009; Bassett et al., 2011). Importantly, changes in connectivity between different mental states have been linked to task performance (Hermundstad et al., 2014), suggesting that the state-dependence of functional connectivity might be an important factor in uncovering the relation between cognition and brain function.

In the current study, we aimed to characterize both the stable, trait-like component of functional connectivity, and the transient, state-dependent component. To this end, we examined functional connectivity in a large population-derived sample of 587 participants, approximately uniformly distributed between 18 and 88 years of age, across three different mental states: eyesclosed resting state, movie watching, and a simple sensorimotor response task. Whereas previous studies (Krienen et al., 2014; see also Cole et al., 2014) have compared commonalities and differences across mental states in the "average" functional connectivity across individuals, we examined how the pattern of individual differences, such as those related to age, varied with state. This is important, because two states could have the same average functional connectivity, yet have a different rank ordering of individuals in terms of their connectivity.

In our analysis of "global" trait and state effects, we used an approach that was used previously to demonstrate the similarity of the average network architecture across states (Cole at al., 2014). This method tests the correlation between connectivity matrices: matrices that contain estimates of connection strengths between every pair of brain regions. We extended previous findings by quantifying the contribution of trait and state effects to individual differences in this regional connectivity (as well as to average connectivity across individuals).

Most studies of functional connectivity do not focus on overall similarity in connectivity, but instead examine the strength of one or more specific connections between regions or networks. In our second, more "local" analysis, we examined how specific

David Troy; Affiliated Personnel: Tibor Auer, Marta Correia, Lu Gao, Emma Green, Rafael Henriques; Research Interviewers: Jodie Allen, Gillian Amery, Liana Amunts, Anne Barcroft, Amanda Castle, Cheryl Dias, Jonathan Dowrick, Melissa Fair, Hayley Fisher, Anna Goulding, Adarsh Grewal, Geoff Hale, Andrew Hilton, Frances Johnson, Patricia Johnston, Thea Kavanagh-Williamson, Magdalena Kwasniewska, Alison McMinn, Kim Norman, Jessica Penrose, Fiona Roby, Diane Rowland, John Sargeant, Maggie Squire, Beth Stevens, Aldabra Stoddart, Cheryl Stone, Tracy Thompson, Ozlem Yazlik; and administrative staff: Dan Barnes, Marie Dixon, Jaya Hillman, Joanne Mitchell, Laura Villis.

Correspondence should be addressed to Linda Geerligs, MRC Cognition and Brain Sciences Unit, 15 Chaucer Road, Cambridge, CB2 7EF, UK. E-mail: lindageerligs@gmail.com.

DOI:10.1523/JNEUROSCI.1324-15.2015

Copyright $\odot 2015$ the authors $\quad 0270-6474 / 15 / 3513950-13 \$ 15.00 / 0$ connections are affected by state, by age, and by interactions between state and age. To improve the interpretability of the results, we performed this analysis at the level of functional networks, focusing on connections within and between networks. Despite the differences in our global and local analyses, results of both suggest that studying individual differences within one state (such as resting) discloses only part of the relevant individual differences in brain function, and that the study of functional connectivity under multiple mental states is essential to disentangle connectivity differences that are transient versus those that represent more stable, trait-like characteristics of an individual.

\section{Materials and Methods}

Participants. A sample of 632 participants (18-88 years old; mean, 54.1; $\mathrm{SD}, 18.5 ; 312$ males and 320 females) were taken from the populationderived " 700 " sample of the Cambridge Centre for Aging and Neuroscience (Cam-CAN). Participants were approximately equally distributed across the lifespan, with the exception of the first decade, which contained fewer participants ( $18-28$ years, 50 participants; $28-38$ years, 104; $38-48$ years, $97 ; 48-58$ years, 96 ; $58-68$ years, $98 ; 68-78$ years, 98 ; $78-88$ years, 89 ). Participants were included if no brain abnormalities were detected, and if they completed the (f)MRI testing session. Participants had no contraindications to MRI, were native English-speakers, had normal or corrected-to-normal vision and hearing, scored 25 or higher on the mini mental state exam (MMSE; Folstein et al., 1975), and had no self-reported history of drug or alcohol abuse, or of neurological disorders (for further details, see Shafto et al., 2014). The study was approved by the Cambridgeshire 2 Research Ethics Committee, United Kingdom. Informed consent was obtained from all participants.

fMRI data and image acquisition. fMRI data were recorded in three different mental states: resting state, a sensorimotor task, and movie watching. These states lasted $\sim 8.5 \mathrm{~min}$ and were recorded in a fixed order, with resting state first, sensorimotor task second, and movie watching last. During the resting state, participants were instructed to close their eyes and not think of any one thing in particular. In the sensorimotor task, participants were instructed to respond by pressing a button with their right index finger when they heard a bilateral $300 \mathrm{~ms}$ tone (at 300,600 , or $1200 \mathrm{~Hz}$ ) and/or simultaneously saw a bilateral checkerboard pattern, presented for $34 \mathrm{~ms}$. A total of 121 trials were presented, with stimulus onset asynchronies ranging from 2 to $26 \mathrm{~s}$. The movie task was used to assess neural connectivity under conditions of continuous visual and auditory input, as participants watched an excerpt of a compelling but unfamiliar film (Shafto et al., 2014).

MRI data were collected as part of a $1 \mathrm{~h}$ session in a 3T Siemens TIM Trio, with a 32 channel head-coil. For resting state, as well as the sensorimotor task, 261 volumes were acquired, each containing 32 axial slices (acquired in descending order), with slice thickness of $3.7 \mathrm{~mm}$ and interslice gap of $20 \%$ (for whole-brain coverage including cerebellum; TR = $1970 \mathrm{~ms} ; \mathrm{TE}=30 \mathrm{~ms}$; flip angle $=78^{\circ} ; \mathrm{FOV}=192 \mathrm{~mm} \times 192 \mathrm{~mm}$; voxel size $=3 \mathrm{~mm} \times 3 \mathrm{~mm} \times 4.44 \mathrm{~mm}$ ) and acquisition time of $8 \mathrm{~min}$ and $40 \mathrm{~s}$. During the movie watching, 193 volumes were acquired with the same slice profile, but using a multi-echo, $\mathrm{T} 2^{*}$-weighted EPI sequence $[\mathrm{TR}=$ $2470 \mathrm{~ms}$, five echoes (TE $=9.4 \mathrm{~ms}, 21.2 \mathrm{~ms}, 33 \mathrm{~ms}, 45 \mathrm{~ms}$, $57 \mathrm{~ms}$ ), flip angle $=78^{\circ}$ ] with an acquisition time of $8 \mathrm{~min}$ and $13 \mathrm{~s}$. The multi-echo data were combined by computing an average of the 5 echo times, weighted by their estimated $\mathrm{T} 2{ }^{\star}$ contrast. Higher-resolution $(1 \mathrm{~mm} \times 1$ $\mathrm{mm} \times 1 \mathrm{~mm}$ ) T1- and T2-weighted structural images were also acquired (to aid registration across participants; Taylor et al., 2013).

Data preprocessing. Preprocessing was performed with the SPM 12 software (http://www.fil.ion.ucl.ac.uk/spm), using the automatic analysis (AA) batching system (http://imaging.mrc-cbu.cam.ac.uk/imaging/ A A). For each participant, field maps were used to undistort the functional EPI images. Subsequently, the functional images were motion-corrected and slice-time corrected. The T1 and T2 structural images were coregistered to an MNI template image, bias-corrected, and then combined to segment various tissue classes (Taylor et al., 2013) using unified segmentation (Ashburner and Friston, 2005). The gray matter and white matter (WM) segments for each participant were used 
to create a study-specific anatomical template, using the DARTEL procedure to optimize inter-participant alignment (Ashburner, 2007), which was subsequently transformed into MNI space. The EPI images were then coregistered to the T1 image, and the DARTEL flow fields and MNI transformation were applied to the EPI images. The segmented images were also used to create WM and CSF masks for each participant by selecting only voxels with $<1 \%$ of gray matter and $>80 \%$ of WM/CSF.

Functional connectivity analysis and motion correction. To reduce the effects of motion on the functional connectivity results, we used a combination of approaches. The first of these was to apply the wavelet despike method for removing motion artifacts from fMRI data without the need for data scrubbing (Patel et al., 2014). The method detects irregular events at different frequencies by identifying chains of outlying wavelet coefficients, and projects these out of the voxel time series. The algorithm can remove both prolonged motion artifacts, such as spin-history effects, and higher-frequency events, such as spikes. The total amount of despiking performed on a dataset is quantified by the percentage of voxels containing a spike in that volume of data.

To quantify the total motion for each participant, the root mean square volume-to-volume displacement was computed using the approach of Jenkinson et al. (2002). Participants with an average spike percentage, in any of the mental states, of 2 SDs above the mean across all mental states $(6.75 \%)$ were excluded from further analysis. This led to the exclusion of 45 datasets ( $7 \%$ of participants). After the wavelet denoising step, the data were smoothed with an $8 \mathrm{~mm}$ FWHM Gaussian kernel.

After smoothing, data were extracted for each of 840 functionally defined regions of interest (ROIs) created by Craddock et al. (2012). To ensure that we only included ROIs that did not suffer from susceptibility artifacts, participant-specific brain masks were created by thresholding functional images at $70 \%$ of mean signal intensity. The 92 ROIs that had an overlap of $<50 \%$ with this mask, in one or more participants and in one or more mental states, were excluded from further analysis (leaving 748). For a control analysis, we used a different parcellation scheme, consisting of 264 ROIs created by Power et al. (2011), of which 227 remained after removing regions with insufficient coverage with our functional scans.

The second step to reduce the effects of motion and other noise confounds on functional connectivity results was to apply multiple regression. The regression of data from one ROI on another included simultaneous regression of expansions of the six original motion parameters, as well as of average signals in the WM and CSF. The WM and CSF signals were created by averaging across voxels in the associated mask image, after the wavelet despiking (but before smoothing). The expansions included the first-order temporal derivative, as well as their squares and squared derivatives, which recent research has shown reduces the effects of motion (Satterthwaite et al., 2013). In total, there were 32 confound and noise regressors. In addition, bandpass filtering from 0.009 to $0.1 \mathrm{~Hz}$ was implemented by regressing out a discrete cosine transform set, consisting of 168 regressors for the rest and sensorimotor data and 123 regressors for the movie data. As an estimate of the connectivity strength of each pair of ROIs, we used the $z$ values resulting from this multiple-regression model, averaged across each ROI, in the pair appearing as a dependent and independent variable.

Because we observed a significant positive correlation between relative displacement and age (resting, $r=0.43$; sensorimotor, $r=0.46$; movie, $r=0.51$ ), we applied a final correction for motion at the group level. This was done by regressing out, for each mental state separately, the mean relative displacement from the connectivity values of each ROI pair (Yan et al., 2013).

Based on the functional connectivity matrices of all participants and mental states, we defined a set of functional networks using a consensus partitioning algorithm (Lancichinetti and Fortunato, 2012). Before the partitioning, all nonsignificant connectivity values $(z<1.96)$ were set to zero, as well as connections between ROIs $<20 \mathrm{~mm}$ apart (Power et al., 2011). An initial partition into functional networks was created using the Louvain modularity algorithm (Blondel et al., 2008), and this partition was refined using a modularity fine-tuning algorithm (Sun et al., 2009). This partitioning was repeated 50 times, and all repetitions were then combined into an ROI-by-ROI consensus matrix. Each element in the consensus matrix indicates the proportion of repetitions in which the corresponding two ROIs were assigned to the same cluster. This matrix was then used as the input for a new partitioning, until the algorithm converged to a single partition (such that consensus matrix consisted only of ones and zeroes). The partitions of all participants and all mental states were combined in a group consensus matrix partitioned using the same consensus algorithm. The procedure described above was applied for multiple resolutions (varying gamma between 1 and 3; Reichardt and Bornholdt, 2006). The most stable partitioning (highest normalized mutual information between solutions at different resolutions) was used as our final set of functional networks (gamma $=2.6$ ). In addition to the 16 large networks described in the main text, we identified five small networks, with $<8$ nodes each. These were excluded from analyses, because their number of ROIs was too small for reliable network connectivity measures.

Global state and trait effects. For the first analyses of state and trait effects on global patterns of functional connectivity, we used a slightly different estimate of functional connectivity. Instead of $Z$-statistics, we computed the Pearson correlations between each pair of ROIs, using the residuals of the regression model described above (i.e., after adjusting for movement-related confounds). This was necessary because we needed multiple connectivity estimates for different parts of the data (see below). Correlations were transformed to $Z$-scores (Fisher, 1921), which resulted in a $748 \times 748$ symmetrical, "ROI connectivity" matrix, calculated separately for each state and each participant.

As a starting point, we performed an analysis similar to that of Cole et al. (2014), in which we averaged the connectivity matrices across participants within each state, and then calculated the spatial correlation across all ROI-pairs to index the similarity of the connectivity patterns across states. The square of this between-state correlation estimates the proportion of variance shared between states. The remaining variance is a combination of state differences and random noise.

We can therefore estimate the variance explained by state effects as the proportion of variance left after subtracting the variance shared across states and the variance due to noise. To estimate the random noise component, we calculated the similarity of connectivity matrices within states. The time-series of each state were split into two halves, with 5 TRs in between the halves, and separate connectivity matrices computed for each half. These connectivity matrices were then averaged across participants, and the correlation between the resulting connectivity matrices of the two halves was computed. This average, within-state, withinindividual correlation is expected to be high, barring the effect of noise. The proportion of variance due to noise was thus estimated as 1 minus this correlation squared. For each comparison between states (e.g., rest versus movie), we used the minimum of the within-state correlations across those two states to provide a liberal estimate of noise (and hence a conservative estimate of state effects). Note that the split-half estimates of similarity within-state arise from half as many time points as the similarity estimates across states, and so are likely to underestimate within-state similarity, while any temporal dynamics of functional connectivity within a given state would also reduce estimates of within-state similarity. In both cases, this would again lead to an overestimate noise, and thereby a conservative estimate of state effects.

The procedure described above investigates the similarities and differences of average functional connectivity matrices. In a similar way, we can investigate whether an individual difference, such as age, is largely driven by trait effects (similar across states) versus state effects (different between states). First, for each state, we computed the correlation between age and functional connectivity for each ROI-pair. This provides us with a matrix of age effects on functional connectivity for each state. Similar to the previous analysis, we compared these matrices between states to test whether pairs of ROIs that show a strong effect of aging on connectivity in one state also show strong effects in a different state. Subsequently, the split-half procedure described above was used to estimate the expected variation in the age effects due to noise.

To extrapolate this to a more general measure of any individual difference, we performed a final global analysis. Here, we first measured the similarity between each pair of participants by correlating their functional connectivity matrices. We did this for each state separately, result- 
ing in a participant-by-participant (rather than ROI-by-ROI) matrix for each state, indicating the similarity in functional connectivity patterns between each pair of participants. Subsequently, we correlated these similarity matrices between states. As above, we used split-half analyses to estimate the variance due to noise.

Because the estimate of random noise is crucial for our estimate of state effects, we compared our split-half estimates to an alternative noise estimate. For this, we concatenated the normalized ( $z$-scored) time series for the two states used in each pairwise comparison across states. Before concatenation, the time series of the movie data were interpolated to the same sampling rate as the rest and sensorimotor data. The interpolation did not alter the results, as the estimates of the overlap between states were identical for the interpolated and the original data. Then, we applied phase randomization to remove any true differences in functional connectivity between the two states. For phase randomization, the data were converted to the frequency domain using a fast Fourier transformation. Although the power spectrum is left intact, the phases are replaced by random phases which are generated separately for each frequency (Prichard and Theiler, 1994). The same set of random phases was applied to the time series of all ROIs. Data were then transformed back into the time domain and connectivity for both states was recomputed (i.e., the first and second half of the concatenated data).

Phase randomization preserves the mean, variance, and the average autocorrelation of the time series, while randomizing the precise timing. Therefore, any true differences in connectivity between the first and the second part of the time series (corresponding to two states) are removed. Similarity of average functional connectivity, age effects, or individual differences was then recomputed as described above. Repeated randomizations gave highly similar estimates for the size of the state effects, suggesting that the estimates were very stable. In Results, we report the minimum value of the observed state effects across 100 repetitions of the phase randomization. The estimates of state effects were consistently larger than the estimates we obtained from the split-half analysis above for each of the state comparisons. Phase randomization is a less conservative estimate of state effects, because it is not affected by true changes in connectivity over time and it uses the same number of data points as the real estimates of between-state similarities. However, phase randomization might overestimate the state effects if there is a difference in noise levels between states.

Local state and trait effects. In our second main set of analyses, we tested how the effects of state on individual differences can alter the conclusions of a typical functional connectivity study. To this end, we estimated the main effect of state, the main effect of age, and the interaction of state and age at the level of individual connections. These connections were estimated at the level of networks, instead of ROIs, to reduce the number of multiple comparisons and to improve the interpretability of the results.

We observed that the average functional connectivity across all connections, was reduced with age in all three cognitive states (rest, $r=$ $-0.28, p<0.001$; sensorimotor, $r=-0.16, p<0.001$; movie, $r=-0.15$, $p<0.001)$. Therefore we, we applied proportional thresholding, in which we kept only the $10 \%$ strongest connections for each participant in each state. Subsequently, we binarized all connections (i.e., set all suprathreshold connections to one and all others to zero) and computed the average within- and between-network connectivity. This ensures that all participants possess the same number of connections, and that only the structure of the network was relevant, regardless of variations in the absolute strength of connections across participants. Subsequently, the ROI connections within or between each of the 16 networks were averaged, to produce a $16 \times 16$ symmetrical network connectivity matrix (Geerligs et al., 2015). For each of the connections within this matrix, we examined changes in connectivity between mental states, using the Wilcoxon signed rank test. Effects of age on connectivity and age-by-state interactions were tested using Spearman correlations.

\section{Results}

Previous research has shown that the average functional network structure across individuals is highly similar across a range of tasks (Cole et al., 2014; Krienen et al., 2014). In the current study, we aimed to assess whether differences between individuals in their functional architecture are also task invariant. This distinction is important, because two states could have the same average functional connectivity, yet a completely different rank ordering of individuals in terms of their connectivity. For example, younger people might have greater functional connectivity than older people in one state, but older people might have relatively greater connectivity in a different state.

To allow for functional interpretation of the connectivity matrices, we identified functional networks across all participants and states, using a two-step multi-resolution consensus community-detection algorithm (Reichardt and Bornholdt, 2006; Blondel et al., 2008; Lancichinetti and Fortunato, 2012). The resulting 16 networks (Fig. $1 A$ ) were highly similar to those in previous studies, whether they used similar graph theoretical approaches or independent component analyses (Beckmann et al., 2005; Power et al., 2011).

Our main analyses focused on comparisons between the resting state and sensorimotor task, because these tasks had identical scanning parameters, ensuring that any differences observed between states were driven only by the cognitive states. The movie data were acquired with a different sequence and are therefore used only to provide supporting evidence for our claims.

\section{Average global connectivity across individuals}

To ground our results in previous work, our first analysis aimed at replicating the work of Cole et al. (2014), using similar methods. First, we computed an average connectivity matrix across all participants for each state (rest, sensorimotor task, and movie). Figure $1 B$ shows that average connectivity was strongest in resting state (mean, 0.17; SD, 0.18), weaker in the sensorimotor task (mean, 0.16; SD, 0.18), and weakest in the movie ( $\mathrm{M}, 0.14$; SD, $0.16)$. We then used spatial correlation across ROIs to index the similarity of the connectivity patterns across states. We observed that average functional architecture was highly similar in the resting state and sensorimotor task $(r=0.93)$. The correlations between rest and movie, and between sensorimotor and movie, were lower $(r=0.80)$, but still in the range of correlations observed by Cole et al. (2014).

To quantify the relative size of the overlap, relative to the differences between states, we compared these correlations to a null-distribution based on the similarity of connectivity matrices within each individual in each state (split-half estimates; see Materials and Methods). This allows an estimate of the amount of variance in the connectivity matrices explained by true state differences. Note that our estimates of such state effects erred on the conservative side (see Materials and Methods), given the relative novelty of these effects. We found that the overlap between the rest and sensorimotor states explained $87 \%$ of the variance, whereas the differences accounted for $12 \%$ of the variance. For the comparisons between rest and movie, and sensorimotor and movie, the overlap was lower $(63 \%$ and $64 \%$, respectively), whereas state differences were larger $(29 \%$ and $36 \%$, respectively).

\section{Individual differences in global connectivity}

Age

Our next step was to go beyond the analysis by Cole et al. (2014), by investigating whether individual differences in functional connectivity are also sensitive to mental state. Instead of focusing on the group average functional connectivity matrix, as above, we investigated the effects of one dimension of individual difference-age- on functional connectivity between each pair of 


\section{A Functional Networks}

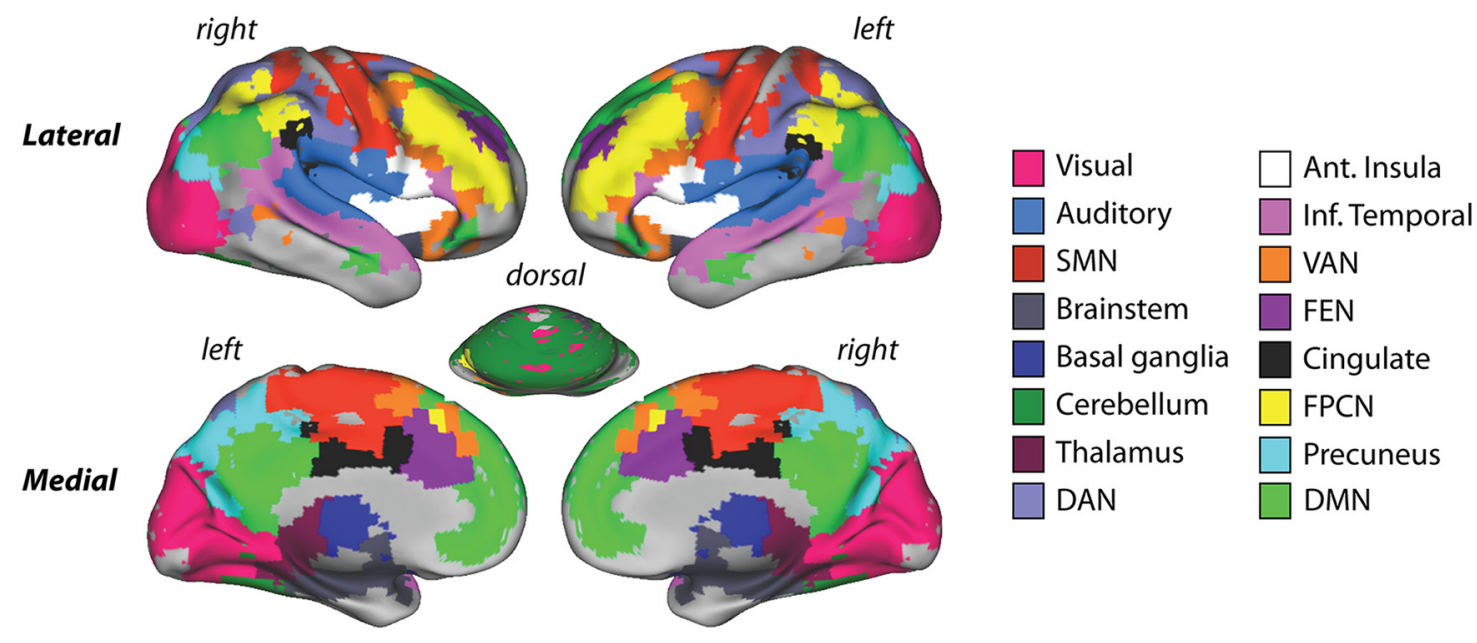

\section{B Average functional connectivity across participants}

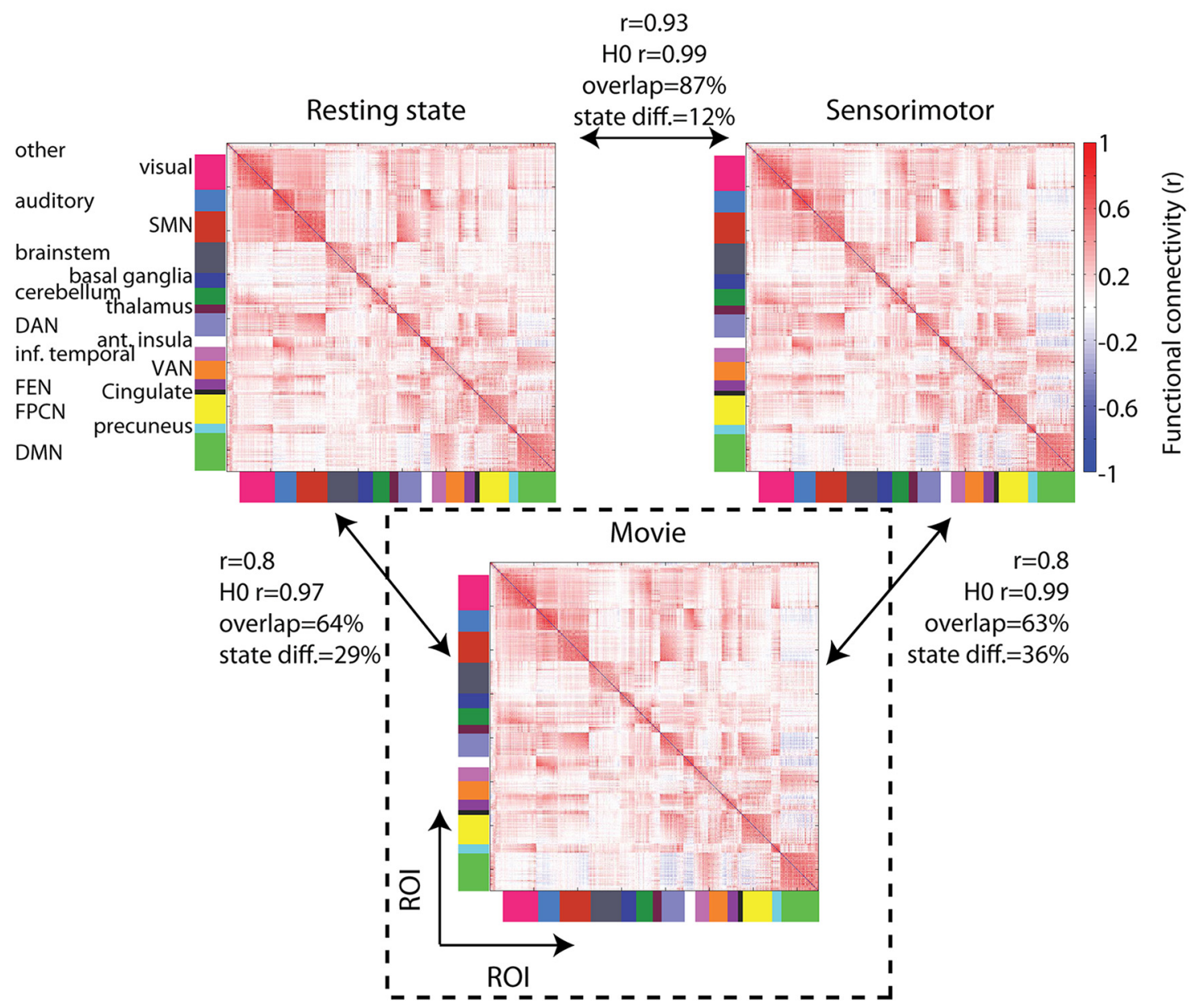

Figure 1. A, The 16 functional networks are displayed on an inflated surface rendering of the human brain using the CARET program (Van Essen et al., 2001). We identified three networks related to sensory and motor processes: the auditory network, the visual network, and the somato-motor network (SMN). In addition, we identified four subcortical networks: a brainstem network, a basal ganglia network, a cerebellar network, and a posterior thalamus network. The remainder were higher-order processing networks, and included the default mode network (DMN), the precuneus network, the anterior insula network, the inferior temporal network, the dorsal attention network (DAN), the ventral attention network (VAN), the fronto-executive network (FEN), and the frontoparietal control network (FPCN). B, An illustration of the average functional connectivity matrices in each mental state. ROls are ordered by functional network, as indicated by the colors on the left side and bottom of the functional connectivity matrices. For each pair of states, the similarity between the connectivity matrices is computed ( $r$ ). The expected value for the similarity if there were no true state difference (based on split-half analyses) is shown by the HO value. Based on these two correlation coefficients, we computed the percentage of variance in the functional connectivity matrix that is shared between states (overlap) and the percentage of variance that is due to state-related changes in connectivity (state diff.), relative to noise (see Materials and Methods). Dashed black box around movie state indicates that the scanning parameters were different from those used in rest and sensorimotor task (see Materials and Methods). 


\section{Relation between age and functional connectivity}

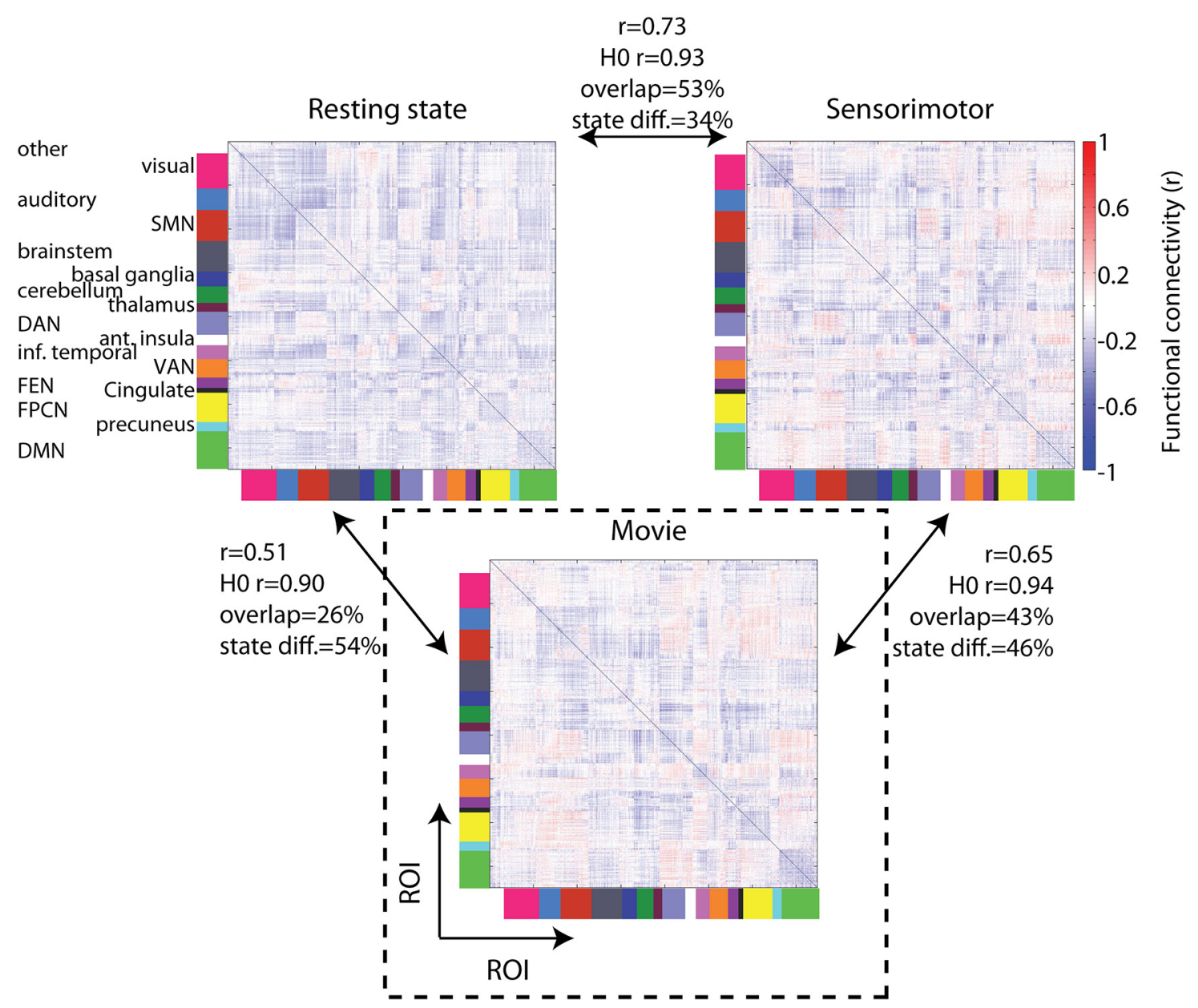

Figure 2. An illustration of the effects of age on functional connectivity matrices in each mental state. The values show the similarity of age effects between states $(r)$, the similarity between states under the null-distribution (H0), the percentage of variance explained by trait effects (overlap), and the percentage of variance explained by state effects based on split-half analyses (state diff.). See Figure 1 legend for more details.

ROIs. We asked whether the pattern of age-related changes that we observe in one state is similar to the pattern of age-related change in other states (i.e., are the connections that decline with age in one state the same connections that show declining connectivity in another state?). In other words, we again computed the similarity between ROI-by-ROI matrices, except that the elements of these matrices now contained the correlation between age and connectivity for each pair of ROIs, rather than the mean connectivity across individuals (Fig. 2).

Looking at the similarities between states in these age effects, we observed an overlap of $42 \%$ for rest versus sensorimotor task, with state differences explaining another $41 \%$ of the variance. It is possible that regressing out average motion inadvertently led to an overestimation of the size of the state effects. Therefore, we repeated this analysis with data in which no group motion correction was applied. This did indeed result in slightly higher estimates for the overlap between states and lower estimates for the state differences (53\% and 34\%, respectively). All the results for data with group motion correction can be found in Table 1, while Figures 2 and 3 are based on data without group motion correction, to provide a conservative estimate of state effects (group motion correction does not affect the results reported in Fig. 1). The group motion correction did not have a large effect on the localization of the age effects, as we observed very high correla- tions between the matrices shown in Figure 2 and the corresponding matrices with group motion correction (rest, $r=0.95$; sensorimotor, $r=0.97$; movie, $r=0.94)$. On average, the correlations between age and functional connectivity are negative in all three states (rest: mean, $-0.1, \mathrm{SD}, 0.12$; sensorimotor: mean, -0.051 , SD, 0.15; movie: mean, -0.045 , SD, 0.14).

Even with the more conservative estimate of state effects, age effects showed substantial differences between rest and sensorimotor states. This finding is further supported by a comparison with the movie state. There, we found that the sensorimotor task showed much more overlap with the movie (43\%) than the resting state $(26 \%)$, with corresponding state effects of $46 \%$ versus $54 \%$, respectively. Given the presence of auditory and visual input in both the movie and the sensorimotor task, but not in resting state, these results suggest that there is a more substantial change in the age effects on functional connectivity when the differences in the nature of two cognitive states are more pronounced. In general, we observe that differences between states are almost as important as similarities between states in shaping the observed age effects. The important implication of this finding is that one would draw quite different conclusions about the effect of aging on functional connectivity depending on the state in which that connectivity was measured. 
Table 1. Estimates of the overlap between states and state-differences, expressed in percentage of variance explained

\begin{tabular}{|c|c|c|c|c|c|c|c|c|c|c|}
\hline \multirow[b]{2}{*}{ Comparison } & \multirow[b]{2}{*}{ Method } & \multicolumn{3}{|c|}{ Overlap } & \multicolumn{3}{|c|}{ Difference } & \multicolumn{3}{|c|}{$\begin{array}{l}\text { Difference/ } \\
\text { Overlap }\end{array}$} \\
\hline & & R-SM & R-M & M-SM & R-SM & R-M & M-SM & R-SM & R-M & M-SM \\
\hline \multirow[t]{5}{*}{ Average FC } & 1. C-SH & 87 & 64 & 63 & 12 & 29 & 36 & 0.14 & 0.45 & 0.57 \\
\hline & 2. C-SH-MR & 87 & 64 & 63 & 12 & 29 & 36 & 0.14 & 0.45 & 0.57 \\
\hline & 3. C-SH-MM & 85 & 63 & 62 & 13 & 29 & 36 & 0.15 & 0.46 & 0.58 \\
\hline & 4. C-PR & 87 & 64 & 63 & 13 & 36 & 37 & 0.15 & 0.56 & 0.58 \\
\hline & 5. $P$ & 84 & 61 & 62 & 15 & 32 & 38 & 0.18 & 0.52 & 0.61 \\
\hline \multirow[t]{5}{*}{ Age effects } & 1. C-SH & 53 & 26 & 43 & 34 & 54 & 46 & 0.64 & 2.08 & 1.07 \\
\hline & 2. C-SH-MR & 42 & 17 & 34 & 41 & 52 & 49 & 0.98 & 3.06 & 1.44 \\
\hline & 3. C-SH-MM & 35 & 11 & 24 & 30 & 43 & 48 & 0.86 & 3.91 & 2.00 \\
\hline & 4. C-PR & 53 & 26 & 43 & 41 & 66 & 51 & 0.78 & 2.54 & 1.20 \\
\hline & 5. P-SH & 47 & 27 & 41 & 38 & 52 & 48 & 0.81 & 1.93 & 1.17 \\
\hline \multirow{5}{*}{$\begin{array}{l}\text { Individual } \\
\text { differences }\end{array}$} & 1. C-SH & 21 & 9 & 19 & 21 & 32 & 23 & 1.00 & 3.56 & 1.21 \\
\hline & 2. C-SH-MR & 19 & 6 & 10 & 19 & 26 & 22 & 1.00 & 4.33 & 2.20 \\
\hline & 3. C-SH-MM & 17 & 11 & 15 & 20 & 27 & 22 & 1.18 & 2.45 & 1.47 \\
\hline & 4. C-PR & 21 & 9 & 19 & 48 & 50 & 46 & 2.25 & 5.32 & 2.42 \\
\hline & 5. P-SH & 20 & 9 & 22 & 22 & 33 & 21 & 1.10 & 3.67 & 0.95 \\
\hline
\end{tabular}

For each comparison that is made in the main text, we show the results of four different analysis approaches. In order these are: (1) the standard split-half analysis as reported in the main text; (2) the results when regressing out motion at the group level; (3) the results using a subset of 147 participants across the lifespan who were matched on average motion; (4) the results using phase-randomization as a null model to estimate the size of state differences; and (5) the results using a different set of ROls by Power et al. (2011). C, Using the set of ROls by Craddock et al. (2012); P, using the set of ROls by Power et al (2011); SH, split-half method; MR, results after regressing out effects of average motion for each connection; MM, using a subset of 147 participants across the lifespan who were motion-matched; $P R$, phase-randomization method.

\section{Generic}

Our next analysis was aimed at verifying that this variability of individual differences between states is not just specific to age, but rather a more general aspect of functional connectivity. We first measured the similarity between each pair of participants by correlating their functional connectivity matrices. We did this for each state separately, resulting in a participant-by-participant (rather than ROI-by-ROI) matrix for each state, indicating how similar functional connectivity patterns were in each pair of participants. We observed that the similarities between participants were highest in the sensorimotor task (mean, 0.43; SD, 0.08), slightly lower in resting state (mean, $0.42 ; \mathrm{SD}, 0.08$ ), and lowest in the movie (mean, 0.4; SD, 0.07). Permutation tests showed that all of these differences between states were highly significant $(p<$ 0.001). The results in Figure 3 suggested that the similarities between participants changed with age; older participants were less similar to each other than younger participants. To test this, we computed the average similarity between each participant and all other participants (by averaging across one of the dimensions of the matrices in Fig. 3), and found a significant negative correlation with age in each of the three mental states (rest $r=-0.27$, $p<0.001$; sensorimotor task $r=-0.41$, movie $r=-0.53, p<$ $0.001)$.

Subsequently, we examined whether similarities between participants in one cognitive state would be consistent with the similarities in a different state (i.e., if two participants show highly similar connectivity patterns in one state, will they also show similar connectivity in another state?). To this end, we correlated these similarity matrices between states (see Fig. 3 for a schematic of the methods and results). Again, we found that individual differences were highly dependent on state. For the comparison of rest and sensorimotor task, the overlap between states (21\% of the variance) was approximately equal to the differences between states $(21 \%)$. Similar to the analysis of the age effects, we observed that the similarity between states was lower for the comparison of rest versus movie (overlap, 9\%; differences, 32\%) than the comparison of sensorimotor versus movie (overlap, 19\%; differences,
$23 \%)$. This again suggests that individual differences change less with state when two states are more similar to each other.

\section{Age and state effects on local connections}

So far, we have considered global similarity of connections across pairs of ROIs, or across pairs of individuals. However, typical studies of functional connectivity often examine how the strength of connectivity between specific ROIs or networks relates to a given dimension of individual differences. Here, we used age as an example dimension of individual differences to show how state effects could influence the conclusions drawn in typical studies of functional connectivity. More specifically, for each connection we tested (1) differences between states (main effect of state), (2) individual differences in age (main effect of age), and most interestingly, (3) the interaction between these state and age effects.

To be able to give a functional interpretation to the results and to reduce the number of multiple comparisons, we performed these analyses at the level of the 16 networks in Figure $1 A$. Before computing average connectivity within and between networks, we applied binary proportional thresholding, in which we kept only the $10 \%$ strongest connections for each participant in each state. This ensures that all participants possessed the same number of connections, and that only the structure of the functional connections was relevant, regardless of variations in the absolute strength of connections across participants.

The average within- and between-network connectivity matrices for each state are shown in Figure 4. Figure $4 A$ shows the strength of all the connections within and between networks in the three mental states, while Figure $4 B$ shows the significant differences in functional connectivity that can be observed between states (connections with a central dot are significant after Bonferroni correction). We will not discuss all of the differences in the figure here, but will mention some highlights. In general, we observed a widespread increase, compared with resting state, in between-network connectivity in the sensorimotor task and, in particular, in the movie. A noticeable exception to this general increase was the significantly reduced connectivity between (and within) sensorimotor networks in the movie and sensorimotor task relative to rest (Fig. $4 B$, top left quadrants). In the sensorimotor task, this reduction was restricted to the visual and somatomotor networks, whereas this also extended to the auditory network in the movie. These connectivity reductions are noteworthy because one might expect the opposite pattern of increased sensorimotor coupling, given that the movie and the sensorimotor task both involve coherent sensorimotor stimulation, which is absent in resting state (see Discussion for further consideration).

Having demonstrated highly significant main effects of state on individual connections within and between networks, we next asked which connections showed a main effect of age. In resting state, the most noticeable effect of age was increased connectivity within and between subcortical networks (Fig. 5A), and a tendency for increased connectivity between subcortical and sensorimotor networks, and between subcortical and higher-order networks. In contrast, in the movie and sensorimotor states, we observed a tendency for decreased connectivity with age between higher-order networks.

Finally, we also found some connections in which there was a significant interaction between age and state effects (Fig. 5B). Most noticeable were changes in age-related effects between sensorimotor and resting states (Fig. 5Bi): connectivity within subcortical networks showed greater age-related increases during 

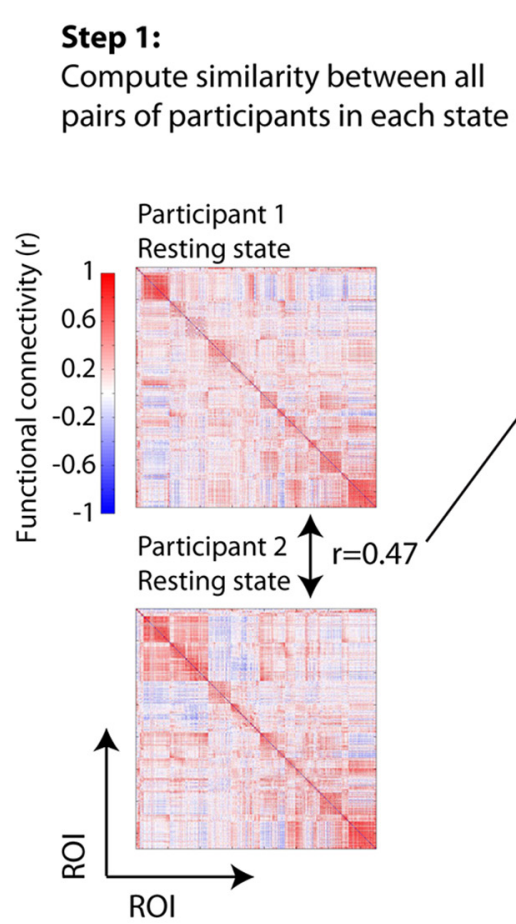

Step 2:

Compare participant similarity

matrices between states
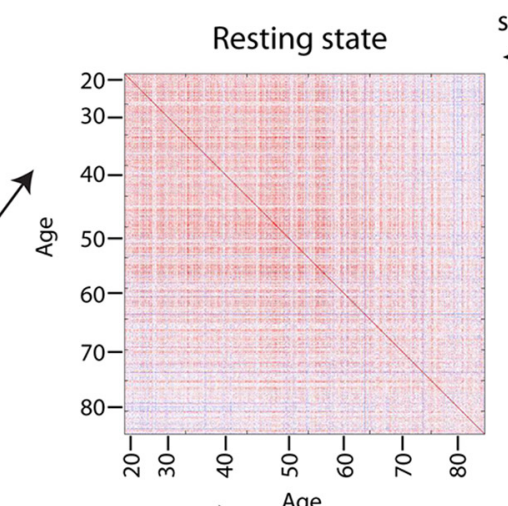

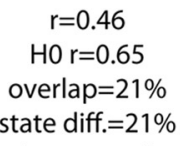

state diff. $=21 \%$
Sensorimotor

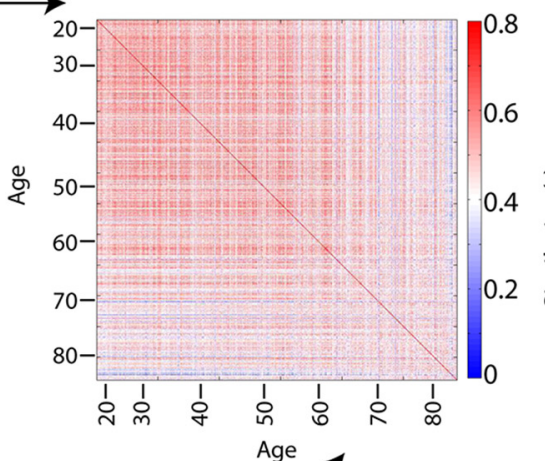

Age

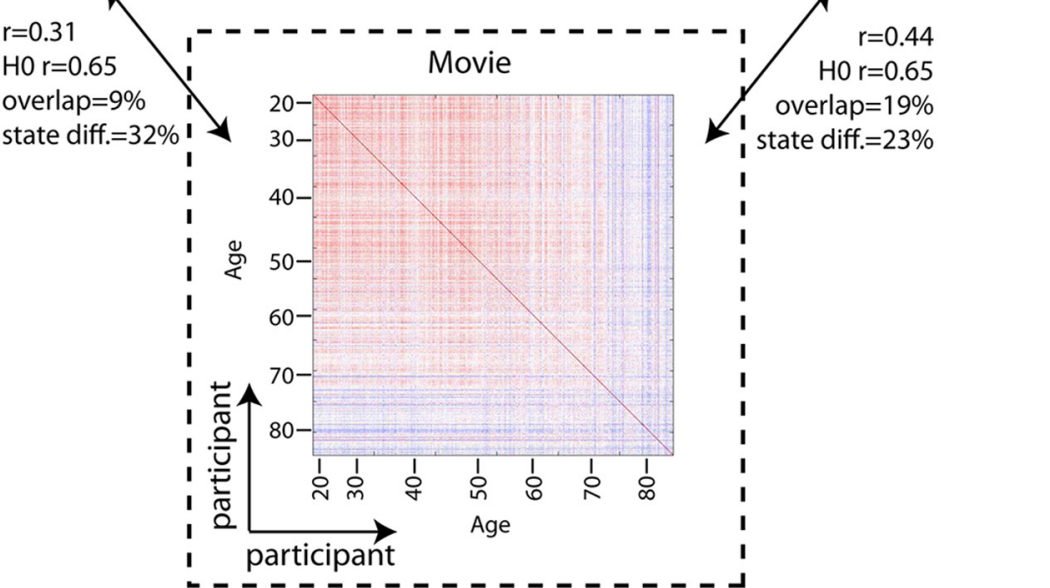

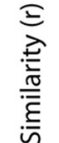

Figure 3. A schematic depiction of the analyses and results of the analysis of individual differences. The first step of the analysis is to compute the correlation between all participants' functional connectivity matrices for each state. This results in a participant-by-participant similarity matrix for each state. Then, the similarity between these participant similarities matrices are computed for each pair of states. Similar to the results in Figures 1 and 2 , the values show the similarity of the participant similarity matrices between states $(r)$, the similarity between states under the null-distribution (HO), the percentage of variance explained by trait effects (overlap), and the percentage of variance explained by state effects based on split-half analyses (state diff.). Note that the participants in the participant similarity matrices are ordered by their age (increasing from top to bottom and from left to right). See Figure 1 legend for more details.

rest, whereas connectivity between subcortical and higher-order networks showed greater age-related decreases during the sensorimotor task (compare Fig. 5Ai, Aii). Also noticeable were changes in the age-related effects within and between sensorimotor networks between movie and rest states (Fig. 5Bii): for example, connectivity between visual and auditory networks showed greater age-related increases in the movie task, whereas connectivity between visual and sensorimotor networks showed greater age-related decreases during rest (compare Fig. 5Aiii, Ai). It is important to note that the differences between the rest and sensorimotor task were more pronounced than the difference between rest and movie and that the differences between the sensorimotor task and the movie were the least prominent. This suggests that these state-by-age interactions are not overly affected by the different acquisition sequence in the movie compared with the rest and sensorimotor states. The important implication of these significant interactions is that the effects of age on specific functional networks would differ across studies that measured connectivity in different states.

\section{Control analyses}

In functional connectivity analyses, motion artifacts are an important concern. This is especially true in aging populations, be- cause in line with previous studies, we observed an age-related increase in average motion for each of the states (resting, $r=0.43$; sensorimotor, $r=0.46$; movie, $r=0.51$ ). We took extra care in our analyses to reduce the effects of motion artifacts on our connectivity estimates (see Materials and Methods). In addition to regressing out quadratic and derivative expansions of the motion parameters, we used wavelet despiking, for which the number of spikes identified was strongly correlated to the average displacement per participant, suggesting that this procedure was successful in some of the age-related differences in motion artifacts $(r=$ 0.74 in resting state, $r=0.67$ in sensorimotor state, $r=0.82$ in movie state). We also removed participants who had excessive numbers of spikes.

To further test the effectiveness of this approach, we examined correlations between functional connectivity estimates and relative displacement. We computed this correlation in 10 year age windows, which we moved across the age range with steps of 1 year to make sure motion and age effects were not confounded. These correlations were averaged across all age groups, after weighting them by the number of participants in each window. We compared our original analysis approach to one in which no wavelet despiking was performed and derivatives and quadratic terms of motion parameters were not regressed out. Two out- 


\section{A Average functional connectivity within and between networks}
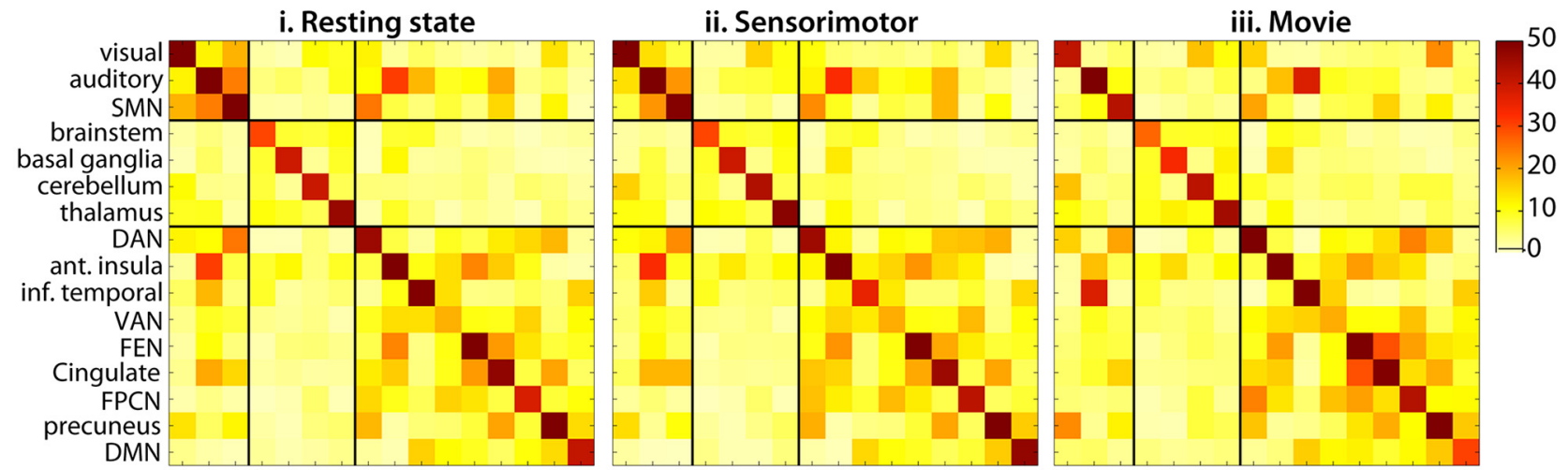

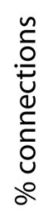

\section{B Differences in functional connectivity between mental states}
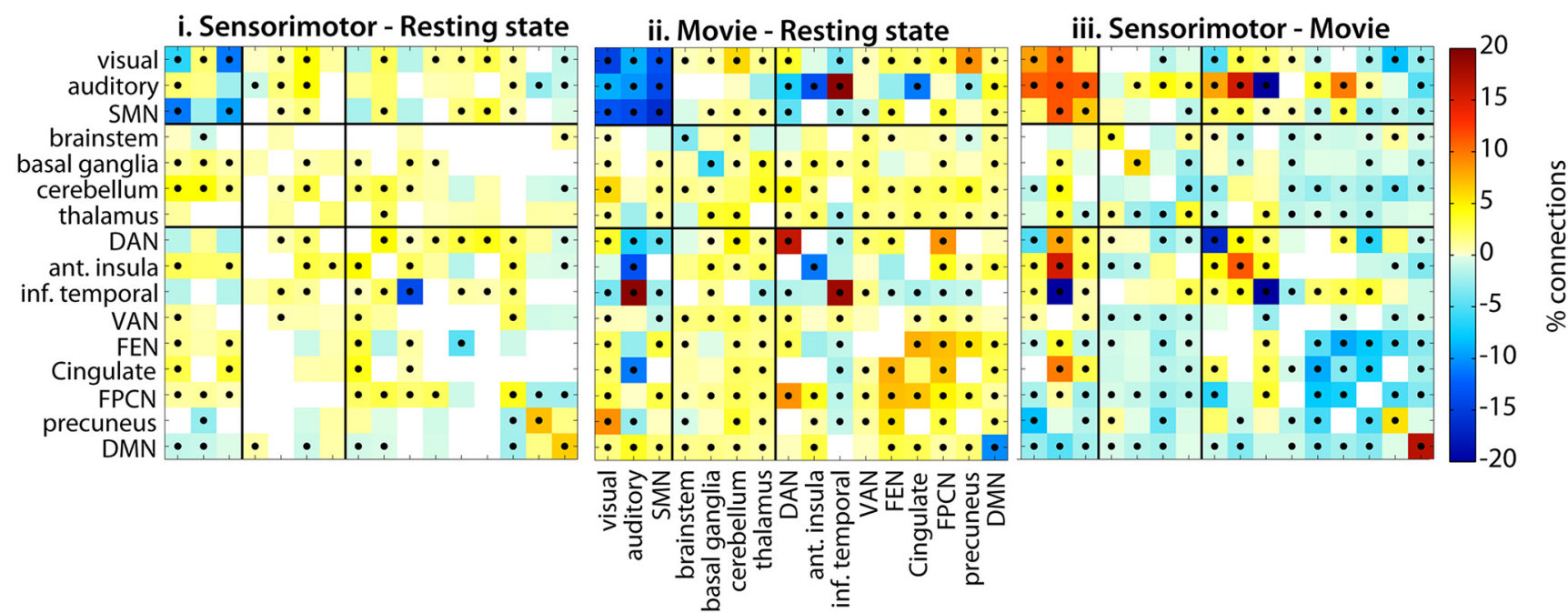

Figure 4. A, Average functional connectivity within and between networks, where lines within each network connectivity matrix illustrate major groupings of networks into "sensorimotor" (i; visual, auditory, SMN), "subcortical" (ii; brainstem, basal ganglia, cerebellum, thalamus), and "higher-order" (iii; DAN, anterior insula, inferior temporal, cingulate, VAN, FEN, FPCN, precuneus, and DMN; see Figure 1 legend for definition of network acronyms). $\boldsymbol{B}$, Differences between mental states, where white squares indicate nonsignificant effects $(p>0.05)$ and black dots indicate effects that remain significant after Bonferroni correction for all multiple comparisons across all connections and states.

come measures that are typically used are the average correlation between connectivity and motion (across distances) and the distance-dependence of these correlations (Patel et al., 2014; Power et al., 2015; given that the effects of motion on connectivity estimates are stronger for pairs of ROIs that are close together). For both measures, we found that the effects of motion were reduced after more elaborate motion correction. The relation between ROI distance and the correlation between motion and functional connectivity reduced in resting state (before $r=-0.067$, after $r=$ -0.057 ), sensorimotor task (before $r=-0.084$, after $r=-0.071$ ), and movie (before $r=-0.075$, after $r=-0.060$ ). In addition, the average correlation with motion was reduced after the elaborate motion correction in resting state (before $r=0.092$, after $r=0.083$ ) and movie (before $r=0.108$, after $r=0.098$ ), though not sensorimotor task (before $r=0.100$, after $r=0.101$ ).

In line with previous work, the results presented above show that there are some residual correlations between motion and functional connectivity, even after elaborate preprocessing (Sat- terthwaite et al., 2013; Yan et al., 2013; Power et al., 2014). This could indicate that motion artifacts were not removed completely. However, it might also be indicative of true differences in functional connectivity between high- and low-motion participants (Zeng et al., 2014). Nevertheless, we wanted to make sure that any age effects we reported could not be due to differences in average motion. This is why we additionally regressed out the average displacement for each participant from each of the functional connectivity estimates (group motion correction).

In addition, we repeated the analyses of global and local connectivity changes in a motion-matched sample of 147 participants across the lifespan. This subsample was created by selecting participants with similar levels of average motion in each decade (correlation between age and average motion in subsample, $r=$ $0.06, p=0.49$ ). Average motion was not regressed out for these analyses. The results of the global connectivity analyses are shown in Table 1 (together with all the other types of control analyses performed here). Because fewer participants are included in the 


\section{A Effects of aging on functional connectivity within and between networks}

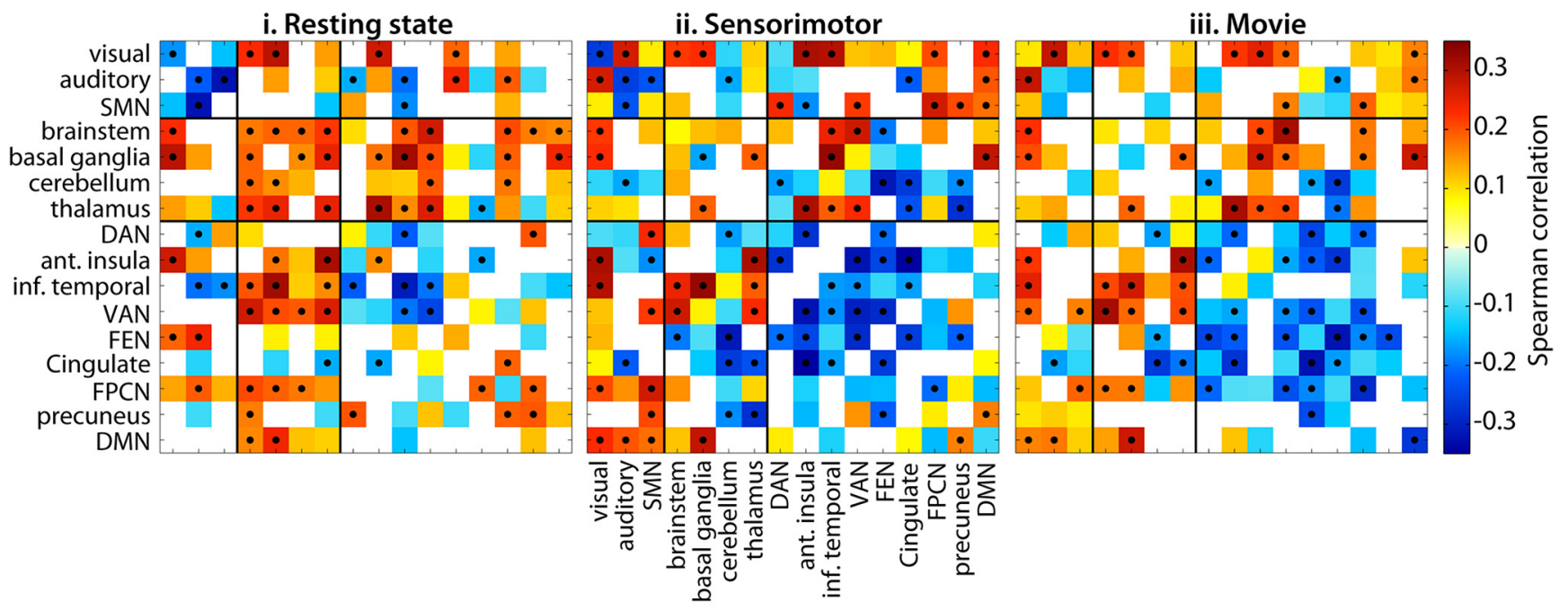

$B$ Differences between states in the effects of aging on functional connectivity
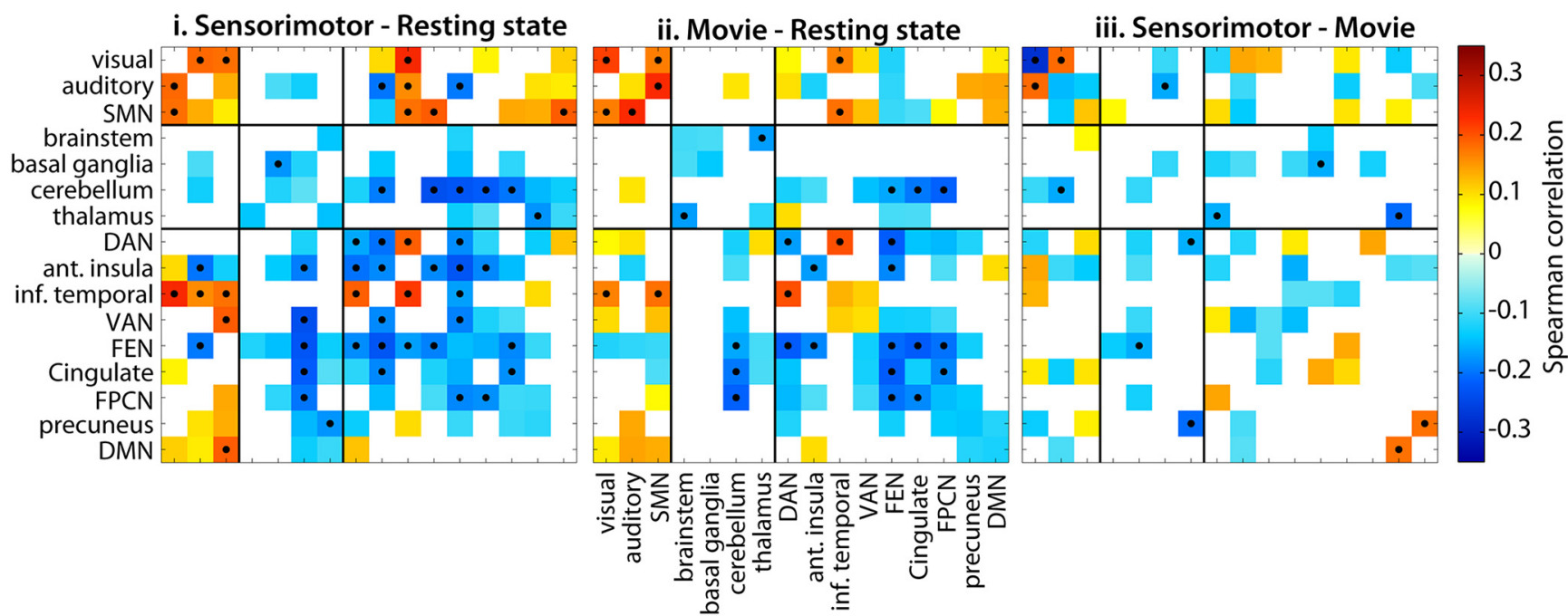

Figure 5. A shows the effects of age on functional connectivity within and between networks within each state separately, while $\boldsymbol{B}$ shows interactions between age and state effects. See Figure 5 legend for more details.

analysis, the estimate of the overlap and the differences between states will be lower than in the original analysis. Nonetheless, considering the ratio between the difference and the overlap between states, it becomes clear that in most cases the state effects are larger in the motion-matched sample than in the full sample, suggesting that motion might be associated with an underestimation of true state effects. This is also confirmed by the results of the local connectivity analysis. In the original analyses in Figure 5, we found that of all the significant associations between age and functional connectivity $(p<0.05), 60 \%$ were correlations between age and connectivity within a given state, while $40 \%$ were age-by-state interactions. This proportion was highly similar in the motion-matched sample (38\%).

As a final check, we investigated in our local connectivity analyses whether there were any motion-by-age interactions that would suggest that the estimated age effect is different for highversus low-motion groups. We found no significant interactions in the resting state or the sensorimotor state after Bonferroni correction. In the movie we did observe two significant interactions, between the precuneus and the cerebellum and between the dorsal attention network and the visual network. For these connections, the effects of age on functional connectivity were positive in the low-motion subgroup and negative in the high-motion subgroup. The low number of significant interactions suggests that interactions between age and motion are not a major confound in our data. If anything, the motion effects might be obscuring some of the true effects of age.

In addition to these checks for motion effects, we examined whether our results were dependent on the specific parcellation used in the analysis. To this end we repeated our analyses, using a parcellation created by Power et al. (2011). The results are reported in Table 1. The estimates of overlap and state effects we obtained with this different parcellation scheme were highly similar to our original results with the Craddock parcellation. In addition, we compared our split-half method for estimating the size of the state effects to an alternative method using phase randomization. Particularly for the analyses of age effects and individual differences, the phase randomization approach led to larger estimates of state effects, suggesting that the split-half method is, if anything, underestimating the true size of the state 
effects due to changes in functional connectivity over time within each state.

\section{Discussion}

Many studies have repeatedly demonstrated the remarkable similarity of functional connectivity patterns across participants and mental states (Calhoun et al., 2008; Smith et al., 2009; Cole et al., 2014; Krienen et al., 2014). In line with this, we observed that average connectivity matrices were highly similar across mental states, with most of the variance (between 63 and 87\%) explained by commonalities in the functional connectivity architecture between states. However, even though the similarities are striking, this does not imply that individual differences in functional connectivity do not vary between mental states. Recent findings concerning the state-dependent nature of functional connectivity suggest that the differences between individuals might vary substantially with their mental state.

In the present work, we disentangled these trait and state aspects of functional connectivity. In the case of aging, one specific dimension of individual difference, we found that state effects were quite substantial when compared with the trait effects (39\% of total explained variance) when comparing resting state to a sensorimotor task. These results show that the regions exhibiting the strongest effects of aging on functional connectivity vary considerably between tasks. When comparing these states with the movie state, we find that the age effects change more when the nature of two states is more dissimilar (e.g., more change between rest-movie than sensorimotor-movie). In a more general analysis of all individual differences, we similarly found that state effects explained as much or more of the variance in the similarity of connectivity matrices between individuals than did trait effects. This suggests that differences between individuals are strongly shaped by the mental state in which these differences are measured, and that both trait and state effects are significant factors in shaping an individual's functional connectivity architecture. Consequently, our ability to understand the changes in brain function that underlie important dimensions of individual differences, such as aging, disorders, or behavioral differences, will be enhanced by considering more than just one mental state.

\section{Effects of age}

We observed that within-network connectivity generally declined with age (Geerligs et al., 2014, 2015), particularly in the movie and sensorimotor task. However, the exact networks that declined varied between mental states. As in previous studies, we observed age-related declines in higher-order networks, such as the default mode network, the frontoparietal control network, and the ventral attention network (Andrews-Hanna et al., 2007; Sambataro et al., 2010). However, in the sensorimotor task, we also observed declines in the auditory and visual networks, which have not been reported previously. Connectivity between networks showed increases as well as decreases with advancing age. In the resting state, for example, we observed increased connectivity between subcortical networks, as well as between subcortical and higher-order networks, supporting the results of Tomasi and Volkow (2012). In contrast, in sensorimotor and movie states, the effects of aging were dominated by a widespread decrease of connectivity between the higher-order networks.

Importantly, there were also connections for which the effects of age depended on state. For example, connectivity within subcortical networks showed greater age effects during rest than during the sensorimotor task, whereas connectivity between dif- ferent higher-order cortical networks showed weaker age effects during rest.

Having said this, it should be noted that the effects of aging were highly dependent on the method of thresholding. When no threshold is applied (as illustrated in Fig. 2), we found that aging was associated with a decline in the majority of within- as well as between-network connections, in each of the mental states. This is due to a shift in the overall distribution of connectivity values with age. It is unclear whether this shift represents a true decrease in neural connectivity, or age-related differences in vascular components of the fMRI signal (D'Esposito et al., 2003; Lu et al., 2011; Tsvetanov et al., 2015). This is why we have focused on the differences in the relative structure of the network (i.e., using proportional thresholding and binarization), which do not depend on overall shifts in the connectivity distribution.

\section{Effects of mental state}

Even though average connectivity architectures were highly similar across mental states, we did observe some interesting differences between states. During the sensorimotor task and movie, compared with rest, we observed increased connectivity between networks, and decreased connectivity within and between the sensorimotor networks. The decreased connectivity within and between sensory networks is noteworthy, because one might expect the continuous, and presumably highly correlated, visual and auditory stimulation in the sensorimotor task and movie to increase connectivity within, as well as between, sensory networks. One possibility is that exogenous stimulation causes greater functional segregation within sensory networks, as specialized components process their preferred dimensions of the stimulus, displacing the highly correlated endogenous activity at rest.

More generally, the similarities of connectivity patterns between different participants depended on mental state: participants were most similar in the sensorimotor task, and least similar in the movie. The sensorimotor task is more constrained than resting, but perhaps also more constrained than watching a movie, e.g., in terms of individual differences in interpretation of that movie. This might make it easier to detect differences between groups of participants, such as patients and controls, in more constrained mental states. However, future research is needed to determine which features of a state elicit high similarity across participants.

\section{Limitations}

Our estimate of percentage of variance explained by true state differences relies on the accuracy of our noise estimates. Our noise estimates were based on the similarity of connectivity patterns in two halves of the same state. Therefore, it was based on fewer time points than our estimate of the similarity between states, which could potentially lead to an underestimation of the percentage of variance explained by the state effects. Nevertheless, we still observed very robust state effects, often of a size similar to that of the trait effects. It is possible, although there are sources of variability that we have not captured with this method. One example is the fixed temporal order of the scanning runs (movie was always last). Participants might have been more tired or less attentive during the movie than in the other mental states, which could have led to an overestimation of the difference between the movie and resting state. In addition, preceding runs may have differential modulation on the functional connectivity in subsequent states. However, such confounds do not undermine the conclusion of this paper: regardless of whether state variability is caused by the stimuli presented, previous tasks, or 
simply time spent in the scanner, these factors modulate individual differences in functional connectivity.

As in all studies of functional connectivity using fMRI, participant motion is an important potential confound. To reduce motion effects, we applied an extensive preprocessing pipeline using state-of-the-art techniques. However, because motion was strongly correlated with age in our sample, this correction may have also removed some neurobiological effects of aging. A further potential confound was that the scanning parameters in the movie differed from those in the other two states (see Materials and Methods). This could have led to an overestimation of the state differences between the movie and other states and, therefore, these state differences should be interpreted with caution. Nonetheless, it is noteworthy that the local interactions between state and age that we found were more pronounced between the rest and sensorimotor sessions (with same scanning parameters) than between these sessions and the movie. This suggests that in this analysis, the effects of state were larger than any effects of scanning parameters.

\section{Conclusion}

The study of functional connectivity in the human brain, in resting as well as task states, has contributed to our understanding of brain function in many ways. However, the results described here show that it is important to keep in mind that differences in functional connectivity between individuals are not static: different patterns of individual differences emerge under different mental states. This is not a flaw of functional connectivity research, but an intriguing characteristic about the way the brain functions. Researchers are beginning to realize that functional connectivity is continuously changing, across periods of seconds, minutes, days, and years, as well as across mental states, and that these changes in turn differ across individuals. Future research should take into account that functional connectivity partly reflects a stable trait of participants, but also partly reflects the mental state of participants, which changes over time. By studying functional connectivity across a wider range of mental states, we might gain a better understanding of the changes in brain function that underlie important dimensions of individual differences, such as aging, disorders, or behavioral differences.

\section{References}

Alexander-Bloch AF, Gogtay N, Meunier D, Birn R, Clasen L, Lalonde F, Lenroot R, Giedd J, Bullmore ET (2010) Disrupted modularity and local connectivity of brain functional networks in childhood-onset schizophrenia. Front Syst Neurosci 4:147. CrossRef Medline

Allen EA, Damaraju E, Plis SM, Erhardt EB, Eichele T, Calhoun VD (2014) Tracking whole-brain connectivity dynamics in the resting state. Cereb Cortex 24:663-676. CrossRef Medline

Andrews-Hanna JR, Snyder AZ, Vincent JL, Lustig C, Head D, Raichle ME, Buckner RL (2007) Disruption of large-scale brain systems in advanced aging. Neuron 56:924-935. CrossRef Medline

Ashburner J (2007) A fast diffeomorphic image registration algorithm. Neuroimage 38:95-113. CrossRef Medline

Ashburner J, Friston KJ (2005) Unified segmentation. Neuroimage 26:839851. CrossRef Medline

Barnes A, Bullmore ET, Suckling J (2009) Endogenous human brain dynamics recover slowly following cognitive effort. PLoS One 4:e6626. CrossRef Medline

Bassett DS, Wymbs NF, Porter MA, Mucha PJ, Carlson JM, Grafton ST (2011) Dynamic reconfiguration of human brain networks during learning. Proc Natl Acad Sci U S A 108:7641-7646. CrossRef Medline

Beckmann CF, DeLuca M, Devlin JT, Smith SM (2005) Investigations into resting-state connectivity using independent component analysis. Philos Trans Biol Sci 360:1001-1013. CrossRef Medline

Blondel VD, Guillaume JLL, Lambiotte R, Lefebvre E (2008) Fast unfolding of communities in large networks. J Stat Mech Theory Exp P10008.
Brier MR, Thomas JB, Fagan AM, Hassenstab J, Holtzman DM, Benzinger TL, Morris JC, Ances BM (2014) Functional connectivity and graph theory in preclinical Alzheimer's disease. Neurobiol Aging 35:757-768. CrossRef Medline

Calhoun VD, Kiehl KA, Pearlson GD (2008) Modulation of temporally coherent brain networks estimated using ICA at rest and during cognitive tasks. Hum Brain Mapp 29:828-838. CrossRef Medline

Cole MW, Bassett DS, Power JD, Braver TS, Petersen SE (2014) Intrinsic and task-evoked network architectures of the human brain. Neuron 83: 238-251. CrossRef Medline

Craddock RC, James GA, Holtzheimer PE 3rd, Hu XP, Mayberg HS (2012) A whole brain fMRI atlas generated via spatially constrained spectral clustering. Hum Brain Mapp 33:1914-1928. CrossRef Medline

Damoiseaux JS, Rombouts SA, Barkhof F, Scheltens P, Stam CJ, Smith SM, Beckmann CF (2006) Consistent resting-state networks across healthy subjects. Proc Natl Acad Sci U S A 103:13848-13853. CrossRef Medline

D'Esposito M, Deouell LY, Gazzaley A (2003) Alterations in the BOLD fMRI signal with ageing and disease: a challenge for neuroimaging. Nat Rev 4:863-872. CrossRef Medline

Di X, Gohel S, Kim EH, Biswal BB (2013) Task vs. rest-different network configurations between the coactivation and the resting-state brain networks. Front Hum Neurosci 7:493. CrossRef Medline

Fisher RA (1921) On the probable error of a coefficient of correlation deduced from a small sample. Metron 1:3-32.

Folstein MF, Folstein SE, McHugh PR (1975) “Mini mental state.” A practical method for grading the cognitive state of patients for the clinician. J Psychiatr Res 12:189-198. CrossRef Medline

Geerligs L, Maurits NM, Renken RJ, Lorist MM (2014) Reduced specificity of functional connectivity in the aging brain during task performance. Hum Brain Mapp 35:319-330. CrossRef Medline

Geerligs L, Renken RJ, Saliasi E, Maurits NM, Lorist MM (2015) A brain wide study of age-related changes in functional connectivity. Cereb Cortex 25:1987-1999. CrossRef Medline

Gonzalez-Castillo J, Hoy CW, Handwerker DA, Robinson ME, Buchanan LC, Saad ZS, Bandettini PA (2015) Tracking ongoing cognition in individuals using whole-brain functional connectivity patterns. Proc Natl Acad Sci U S A 112:8762-8767. CrossRef Medline

Hermundstad AM, Brown KS, Bassett DS, Aminoff EM, Frithsen A, Johnson A, Tipper CM, Miller MB, Grafton ST, Carlson JM (2014) Structurallyconstrained relationships between cognitive states in the human brain. PLoS Comput Biol 10:e1003591. CrossRef Medline

Hutchison RM, Womelsdorf T, Allen EA, Bandettini PA, Calhoun VD, Corbetta M, Della Penna S, Duyn JH, Glover GH, Gonzalez-Castillo J, Handwerker DA, Keilholz S, Kiviniemi V, Leopold DA, de Pasquale F, Sporns O, Walter M, Chang C (2013) Dynamic functional connectivity: promise, issues, and interpretations. Neuroimage. 80:360-378. CrossRef Medline

Jenkinson M, Bannister P, Brady M, Smith S (2002) Improved optimization for the robust and accurate linear registration and motion correction of brain images. Neuroimage 17:825-841. CrossRef Medline

Kitzbichler MG, Henson RN, Smith ML, Nathan PJ, Bullmore ET (2011) Cognitive effort drives workspace configuration of human brain functional networks. J Neurosci 31:8259-8270. CrossRef Medline

Krienen FM, Yeo BT, Buckner RL (2014) Reconfigurable task-dependent functional coupling modes cluster around a core functional architecture. Philos Trans R Soc Lond B Biol Sci 369:pii:20130526. CrossRef Medline

Lancichinetti A, Fortunato S (2012) Consensus clustering in complex networks. Sci Rep 2:336. CrossRef Medline

Lu H, Xu F, Rodrigue KM, Kennedy KM, Cheng Y, Flicker B, Hebrank AC, Uh J, Park DC (2011) Alterations in cerebral metabolic rate and blood supply across the adult lifespan. Cereb Cortex 21:1426-1434. CrossRef Medline

Mennes M, Kelly C, Colcombe S, Castellanos FX, Milham MP (2013) The extrinsic and intrinsic functional architectures of the human brain are not equivalent. Cereb Cortex 23:223-229. CrossRef Medline

Moussa MN, Vechlekar CD, Burdette JH, Steen MR, Hugenschmidt CE, Laurienti PJ (2011) Changes in cognitive state alter human functional brain networks. Front Hum Neurosci 5:83. CrossRef Medline

Patel AX, Kundu P, Rubinov M, Jones PS, Vértes PE, Ersche KD, Suckling J, Bullmore ET (2014) A wavelet method for modeling and despiking motion artifacts from resting-state fMRI time series. Neuroimage 95:287304. CrossRef Medline 
Power JD, Cohen AL, Nelson SM, Wig GS, Barnes KA, Church JA, Vogel AC, Laumann TO, Miezin FM, Schlaggar BL, Petersen SE (2011) Functional network organization of the human brain. Neuron 72:665-678. CrossRef Medline

Power JD, Mitra A, Laumann TO, Snyder AZ, Schlaggar BL, Petersen SE (2014) Methods to detect, characterize, and remove motion artifact in resting state fMRI. Neuroimage 84:320-341. CrossRef Medline

Power JD, Schlaggar BL, Petersen SE (2015) Recent progress and outstanding issues in motion correction in resting state fMRI. Neuroimage 105: 536-551. CrossRef Medline

Prichard D, Theiler J (1994) Generating surrogate data for time series with several simultaneously measured variables. Phys Rev Lett 73:951-954. CrossRef Medline

Reichardt J, Bornholdt S (2006) Statistical mechanics of community detection. Phys Rev E Stat Nonlin Matter Phys 74:016110. CrossRef Medline

Sambataro F, Murty VP, Callicott JH, Tan HY, Das S, Weinberger DR, Mattay VS (2010) Age-related alterations in default mode network: impact on working memory performance. Neurobiol Aging 31:839-852. CrossRef Medline

Sanz-Arigita EJ, Schoonheim MM, Damoiseaux JS, Rombouts SA, Maris E, Barkhof F, Scheltens P, Stam CJ (2010) Loss of "small-world" networks in Alzheimer's disease: graph analysis of FMRI resting-state functional connectivity. PLoS One 5:e13788. CrossRef Medline

Satterthwaite TD, Elliott MA, Gerraty RT, Ruparel K, Loughead J, Calkins ME, Eickhoff SB, Hakonarson H, Gur RC, Gur RE, Wolf DH (2013) An improved framework for confound regression and filtering for control of motion artifact in the preprocessing of resting-state functional connectivity data. Neuroimage 64:240-256. CrossRef Medline

Shafto MA, Tyler LK, Dixon M, Taylor JR, Rowe JB, Cusack R, Calder AJ, Marslen-Wilson WD, Duncan J, Dalgleish T, Henson RN, Brayne C, Matthews FE (2014) The Cambridge Centre for Ageing and Neuroscience (Cam-CAN) study protocol: a cross-sectional, lifespan, multidisciplinary examination of healthy cognitive ageing. BMC Neurol 14:204. CrossRef Medline

Shirer WR, Ryali S, Rykhlevskaia E, Menon V, Greicius MD (2012) Decoding subject-driven cognitive states with whole-brain connectivity patterns. Cereb Cortex 22:158-165. CrossRef Medline
Smith SM, Fox PT, Miller KL, Glahn DC, Fox PM, Mackay CE, Filippini N, Watkins KE, Toro R, Laird AR, Beckmann CF (2009) Correspondence of the brain's functional architecture during activation and rest. Proc Natl Acad Sci U S A 106:13040-13045. CrossRef Medline

Sun Y, Danila B, Josić K, Bassler KE (2009) Improved community structure detection using a modified fine-tuning strategy. Europhys Lett 86:28004. CrossRef

Taylor JM, Correia M, Cusack R, Peelle JE, Cam-CAN, Henson RN (2013) Multichannel (T1, T2, PD) segmentation improves grey matter estimates for voxel-based morphometry (VBM) within and across age groups. Soc Neurosci Abstr 41:679.07.

Tomasi D, Volkow ND (2012) Aging and functional brain networks. Mol Psychiatry 17:549-558. CrossRef Medline

Tsvetanov KA, Henson RN, Tyler LK, Davis SW, Shafto MA, Taylor JR, Williams N, Cam-Can, Rowe JB (2015) The effect of ageing on fMRI: correction for the confounding effects of vascular reactivity evaluated by joint fMRI and MEG in 335 adults. Hum Brain Mapp 36:2248-2269. CrossRef Medline

Uddin LQ, Supekar K, Menon V (2010) Typical and atypical development of functional human brain networks: insights from resting-state FMRI. Front Syst Neurosci 4:21. CrossRef Medline

van den Heuvel MP, Stam CJ, Kahn RS, Hulshoff Pol HE (2009) Efficiency of functional brain networks and intellectual performance. J Neurosci 29:7619-7624. CrossRef Medline

Van Essen DC, Drury HA, Dickson J, Harwell J, Hanlon D, Anderson CH (2001) An integrated software suite for surface-based analyses of cerebral cortex. J Am Med Inform Assoc 8:443-459. CrossRef Medline

Yan CG, Cheung B, Kelly C, Colcombe S, Craddock RC, Di Martino A, Li Q, Zuo XN, Castellanos FX, Milham MP (2013) A comprehensive assessment of regional variation in the impact of head micromovements on functional connectomics. Neuroimage 76:183-201. CrossRef Medline

Zalesky A, Fornito A, Cocchi L, Gollo LL, Breakspear M (2014) Timeresolved resting-state brain networks. Proc Natl Acad Sci U S A 111: 10341-10346. CrossRef Medline

Zeng LL, Wang D, Fox MD, Sabuncu M, Hu D, Ge M, Buckner RL, Liu H (2014) Neurobiological basis of head motion in brain imaging. Proc Natl Acad Sci U S A 111:6058-6062. CrossRef Medline 